\title{
Critical System Cascading Collapse Assessment for Determining the Sensitive Transmission Lines and Severity of Total Loading Conditions
}

\author{
Nur Ashida Salim, ${ }^{1}$ Muhammad Murtadha Othman, \\ Ismail Musirin, ${ }^{2}$ and Mohd Salleh Serwan ${ }^{3}$ \\ ${ }^{1}$ Faculty of Electrical Engineering, Universiti Teknologi MARA, 13500 Pulau Pinang, Malaysia \\ ${ }^{2}$ Faculty of Electrical Engineering, Universiti Teknologi MARA, 40450 Shah Alam, Selangor, Malaysia \\ ${ }^{3}$ Advanced Power Solutions Sdn. Bhd., Worldwide Business Centre, Jalan Tinju 13/50, 40000 Shah Alam, Selangor, Malaysia \\ Correspondence should be addressed to Nur Ashida Salim; ashida_amani@yahoo.com
}

Received 4 April 2013; Revised 19 June 2013; Accepted 7 July 2013

Academic Editor: Yang Tang

Copyright ( 2013 Nur Ashida Salim et al. This is an open access article distributed under the Creative Commons Attribution License, which permits unrestricted use, distribution, and reproduction in any medium, provided the original work is properly cited.

This paper presents a computationally accurate technique used to determine the estimated average probability of a system cascading collapse considering the effect of hidden failure on a protection system. This includes an accurate calculation of the probability of hidden failure as it will give significant effect on the results of the estimated average probability of system cascading collapse. The estimated average probability of a system cascading collapse is then used to determine the severe loading condition contributing to a higher risk of a system cascading collapse. This information is important because it will assist the utility to determine the maximum level of increase in the system loading condition before the occurrence of critical power system cascading collapse. Furthermore, the initial tripping of sensitive transmission line contributing to a critical system cascading collapse can also be determined by using the proposed method. Based on the results obtained from this study, it was found that selecting the accurate probability of hidden failure is very important as it will affect the estimated average probability of a system cascading collapse. Comparative study has been done with other techniques to verify the effectiveness of the proposed method used in the determination of sensitive transmission lines.

\section{Introduction}

In recent years, many blackouts have occurred around the world and the latest blackout had happened in India on the 30th and 31st of July 2012 that affected over 620 million people throughout the country which is estimated to be $9 \%$ of the world population [1]. According to the report of grid disturbance in India [2], the major factors that lead to the initiation of the grid disturbance on the 30th and the 31st of July 2012 were due to weak interconnection between regions. This system was undermined by a number of transmission lines outages connecting the western and northern regions of India. This condition also happened to the Arizona-Southern California Outages on the 8th of September 2011 where the disconnection of a single transmission line was the main reason of the system disturbance that caused approximately
2.7 million populations without electrical power [3]. Other major blackouts afflicted by the system cascading collapse have been reported in $[4,5]$. Normally, cascading outages of transmission lines are the main reason that leads to a large system blackout $[6,7]$. Cascading outage is a sequence of multiple dependent component outages that gradually happened in a power system. Cascading outage is usually caused by an initial failure of a transmission line that propagates to a widespread of system outage.

There are various techniques used to perform the analysis of system cascading collapse. Dobson et al. [5] have applied the branching process model to determine the distribution of cascading outages for a given initial failure. Carreras et al. [7] use the OPA model to identify the overloaded transmission lines with high probability. By applying the OPA model, the proposed technique is capable of recognizing the critical lines 


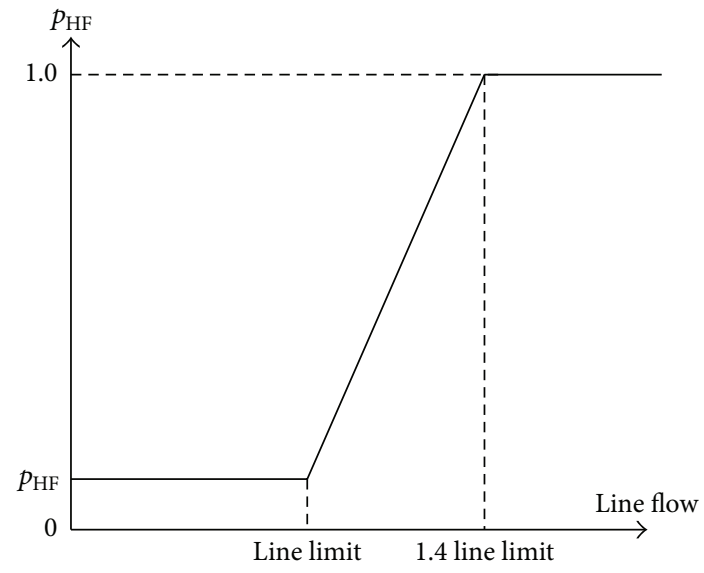

FIGURE 1: Probability of an exposed line incorrect tripping due to the effect of hidden failure.

which contribute to a high probability of cascading collapse. A hybrid approach was proposed by Chen et al. [8] in order to study the structural vulnerability of power networks by using the topological structure error and vulnerability of networks caused by attack from system failure. This technique takes into account the power flow equations and the effect of hidden failure of a protection system. Shi et al. [9] perform the analysis of cascading collapse by considering the hidden failure effect of a protection relay. Wang et al. [10] use the optimal power flow (OPF) technique to identify chains of events occurred in a system cascading outage. The system cascading collapse is performed by taking into account the total power loss reduction which is obtained through the optimum dispatch of active and reactive power generation. Wang et al. [11] use the fault chain theory to perform a system cascading collapse initiated by a transmission line failure. A power flow at several lines is basically used to derive the predictive index which will then be used to instigate subsequent tripping of transmission lines at nonfaulty or normal condition. The process of system cascading collapse is halted once the system instability occurs during the subsequent tripping of transmission lines. Finally, the number of line tripping is basically used to derive the vulnerability index for identifying the sensitive initial line tripping leading to the severity of system cascading collapse.

From the literature review that has been conducted, it is important to study the effect of cascading collapse due to its significant impact on a power system. The report of blackout that has been explained earlier revealed that the main factor that led to a system cascading collapse is due to the initial tripping of transmission line. For that reason, it is crucially important to identify the sensitive transmission lines and also the severity of total loading condition that could initiate system disturbances. Therefore, in the proposed study of cascading collapse, the analysis is carried out to study the impact of different values of hidden failure probability on the estimated average probability of cascading collapse, severity of total loading condition, and sensitive transmission lines. Hidden failure is the main cause of the occurrence of cascading outages which could lead to cascading collapse of a power system. Hidden failure is defined as unobserved deficiency of a protection system that remains hidden due to the anticipation of an unusual system operating condition. In this study, the determination of sensitive transmission lines and severity of total loading condition is performed based on the criticality of the system cascading collapse where the overall procedure can be found in Section 3. The IEEE-RTS79 and IEEE-RTS96 are used as the case studies to validate the effectiveness of the proposed approach considered in the assessment on system cascading collapse. The assessment of cascading events needs to be conducted regularly in the power system operation and planning so that a power system could be prevented from any kind of disastrous events. Therefore, it is important for the utility and power system planner to identify the severe total loading condition and the sensitive transmission lines that will cause significant impact of system cascading collapse.

\section{Probability of Exposed Line Incorrect Tripping Caused by the Hidden Failure}

When a transmission line trips or disconnects from a system, there is a significant probability that the lines connected to either end of the disconnected transmission line might incorrectly trip due to its misoperation of protection relay. These further lines trippings are known as hidden failures as they do not turn out to be noticeable until it appears at the neighboring lines exposed by the initial line tripping.

Relay protection hidden failure is one of the main reasons that led to a system cascading collapse. The probability of exposed line tripping caused by hidden failure should be calculated accurately due to its significant impact on the assessment of system cascading collapse, severe total loading condition, and sensitive transmission lines. As mentioned in Section 1, the hidden failure caused by defective tripping of an exposed line normally starts with an initial tripping of overloaded or faulty line. An initial component tripping may result in a cascading tripping by affecting the neighboring components that will become a contributing factor in spreading the disturbances and finally causing the whole system to collapse. Hidden failures cannot be detected during normal system operating condition. However, when a fault or overloads occur, it will expose the neighboring lines and causing unnecessary outages to the other equipment. The hidden failure leading to an exposed line tripping is selected according to the probability of an incorrect tripping curve, $p_{\mathrm{HF}}$, and this is shown in Figure 1 [12]. Each exposed line has its own load-dependent probability of incorrect tripping, $p_{\mathrm{HF}}$, specifically modeled based on the escalating function of line loading seen by the line protective relay. In Figure 1, the same $p_{\mathrm{HF}}$ values are obtained for a line loading that is equivalent to or lower than the line limit. At the same time, the $p_{\mathrm{HF}}$ increases linearly with regard to the increased in line loading, which is based on the power flow results, until it reaches 1.4 of the line limit.

Based on the excerption taken from NERC report, there were 400 events of cascading collapse caused by the hidden 


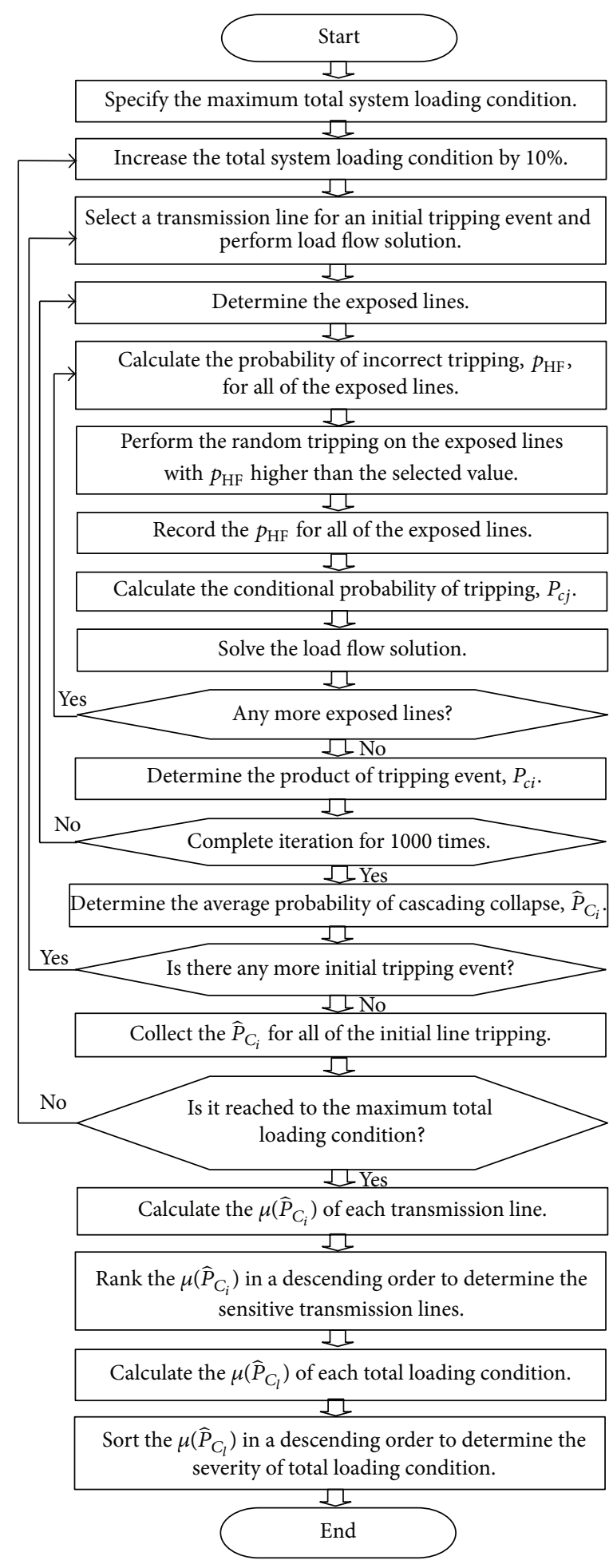

Figure 2: Determination of sensitive transmission lines and severe total loading conditions based on the critical cascading collapse which occurred due to the effect of protection relay hidden failure.

failure of a line protective relay happened in the past 16 years that is from 1984 to 1999 [12]. This means that the probability of occurrence for one exposed line tripping event due to hidden failure is very small but cannot be neglected due to its catastrophic effect on a power system condition.
The probability of one exposed line tripping event due to the hidden failure can be calculated by using (1) as

$$
p_{\mathrm{HF}}=\frac{E}{y \times d \times h \times m \times s} .
$$


For that reason, the probability of an exposed line tripping event due to hidden failure according to the historical information [8] is given by

$p_{\mathrm{HF}}$

$$
\begin{aligned}
& =\frac{400 \text { events }}{16 \text { years } \times 365 \text { days } \times 24 \text { hour } \times 60 \text { minutes } \times 60 \text { second }} \\
& =8 \times 10^{-7} .
\end{aligned}
$$

Then, the probability increases linearly until it reaches $p_{\mathrm{HF}}$ $=1$, that is, 1.4 of its line limit. The probability remains unchanged as $p_{\mathrm{HF}}=1$ for line loading that is above 1.4 of line limit [13], line tripping probability caused by the hidden failure, $p_{\mathrm{HF}}$, since this will assist the utility in providing accurate decision on a power system planning.

\section{Determination of Sensitive Transmission Lines and Severe Total Loading Condition Based on the Critical Cascading Collapse}

This section will discuss the procedures involved to analyze the probability of cascading collapse by taking into consideration three different case studies of hidden failure probability which are $p_{\mathrm{HF}}=8 \times 10^{-7}, p_{\mathrm{HF}}=1 \times 10^{-12}$, and $p_{\mathrm{HF}}=1 \times 10^{-2}$ [13]. For each case study, the results of average probability of cascading collapse will be used to further analyze the severe total loading condition and also to identify the sensitive transmission lines that would cause critical cascading collapse of a power system. The proposed algorithm shown in Figure 2 begins with an initial tripping event of a transmission line. Simultaneously, the power flow solution is performed by taking into account $10 \%$ increase of the total system loading condition. Then, calculate the probability of incorrect tripping, $p_{\mathrm{HF}}$, for each exposed lines connected adjacent to the tripping line.

Perform arbitrary tripping on the exposed lines with $p_{\mathrm{HF}}$ higher than the selected value of $p_{\mathrm{HF}}=8 \times 10^{-7}$. In this case study, $p_{\mathrm{HF}}=8 \times 10^{-7}$ is obtained based on the historical data of transmission line tripping events that caused by the effect of hidden failure and this has been discussed elaborately in Section 2 . This will be used as a benchmark by comparing it with the other case studies which are at the lower end of $p_{\mathrm{HF}}=$ $1 \times 10^{-12}$ and higher end of $p_{\mathrm{HF}}=1 \times 10^{-2}$ in order to observe its significant impact on the probability of cascading collapse, severe total loading condition, and sensitive transmission lines.

Simultaneously, calculate the conditional probability of tripping, $P_{c j}$, [12] by using (3):

$$
P_{c j}=\prod p_{H_{j}} \prod q_{H_{j}}
$$

This process is repeated until there is no exposed line to perform the random tripping event. Then, (4) is used to calculate the tripping events probability, $P_{c i}$, by considering all of the conditional probability of tripping, $P_{c j}$, as

$$
P_{c i}=\prod_{j=1}^{N J} P_{c j} .
$$

For the selected initial line tripping, the simulation is repeated for $K=1000$ times in order to obtain the average probability of cascading collapse, $\widehat{P}_{C_{i}}$, that can be calculated using (5), as

$$
\widehat{P}_{C_{i}}=\frac{1}{K} \sum_{k=1}^{K} P_{C_{i, k}} .
$$

Then, the entire simulation is repeated until the last initial line tripping has been reached. Collect the average probability of cascading collapse, $\widehat{P}_{C_{i}}$, for all of the initial line tripping. Use (6) to calculate the estimated average probability of cascading collapse $\mu\left(\widehat{P}_{C_{i}}\right)$ for identifying the sensitive transmission lines contributing to a critical system cascading collapse as

$$
\mu\left(\widehat{P}_{C_{i}}\right)=\frac{1}{L} \sum_{l=1}^{L} \widehat{P}_{C_{i, l}} .
$$

Rank the $\mu\left(\widehat{P}_{C_{i}}\right)$ in descending order to identify the sensitive transmission lines at initial tripping. The sensitive transmission lines are obtained by referring to the initial line tripping that leads to a sudden increase of $\mu\left(\widehat{P}_{C_{i}}\right)$.

Then, calculate the estimated average probability of system cascading collapse, $\mu\left(\widehat{P}_{C_{l}}\right)$, that is used to identify the criticality of system cascading collapse prior to a severe total loading condition using (7), as

$$
\mu\left(\widehat{P}_{C_{l}}\right)=\frac{1}{I} \sum_{i=1}^{I} \widehat{P}_{C_{i, l}} .
$$

Arrange the $\mu\left(\widehat{P}_{C_{l}}\right)$ in descending order to identify the severity of total loading condition that led to a critical system cascading collapse. The severity of total loading conditions is determined based on the changes of total loading condition that leads to a significant increase of $\mu\left(\widehat{P}_{C_{l}}\right)$.

\section{Results and Discussion}

This section will discuss the estimated average probability of system cascading collapse, $\mu\left(\widehat{P}_{C_{i}}\right)$ and $\mu\left(\widehat{P}_{C_{l}}\right)$, that takes into account the consequence of hidden failure in a relay protection system. The total loading condition is increased by $10 \%$ while maintaining a constant power factor at all buses. An initial tripping event of a transmission line is performed for every increase of total loading condition. The simulation is repeated for 1000 times in order to obtain the $\widehat{P}_{C_{i}}$.

Further analysis was performed on the results of $\mu\left(\widehat{P}_{C_{i}}\right)$ and $\mu\left(\widehat{P}_{C_{l}}\right)$ based on three different cases of hidden failure which are $p_{\mathrm{HF}}=8 \times 10^{-7}, p_{\mathrm{HF}}=1 \times 10^{-12}$, and $p_{\mathrm{HF}}=1 \times 10^{-2}$. 
The $p_{\mathrm{HF}}=8 \times 10^{-7}$ represents the value of probability of hidden failure obtained in accordance with the historical information of a line tripping event caused by the hidden failure. Meanwhile, $p_{\mathrm{HF}}=1 \times 10^{-2}$ used in this analysis is obtained from [9]. Furthermore, the analysis is also performed by considering the $p_{\mathrm{HF}}=1 \times 10^{-12}$ as an additional case study which is lower than the value of $p_{\mathrm{HF}}=8 \times 10^{-7}$ obtained based on the real data. This analysis is performed in order to observe its significant impact on the system cascading collapse when $p_{\mathrm{HF}}$ is smaller than the actual value which is obtained on the historical information.

The IEEE-RTS79 and IEEE-RTS96 are used as the case studies to verify the effectiveness and robustness of the proposed approach considered in the cascading collapse assessment. The data for each system can be found in $[14,15]$, respectively.

4.1. Determination of Sensitive Transmission Lines due to the Effect of Hidden Failure. The estimated average probability of cascading collapse $\mu\left(\widehat{P}_{C_{i}}\right)$ is used to identify the sensitive initial lines tripping which would have high tendency to cause a critical system cascading collapse. By referring to the three case studies of $p_{\mathrm{HF}}$ conducted on the IEEE-RTS79 and IEEE-RTS96, the sensitive transmission lines are obtained by referring to a significantly large value of $\mu\left(\widehat{P}_{C_{i}}\right)$ which indicates the criticality of a system cascading collapse. The $p_{\mathrm{HF}}=8 \times 10^{-7}, p_{\mathrm{HF}}=1 \times 10^{-12}$, and $p_{\mathrm{HF}}=1 \times 10^{-2}$ are the three case studies of probability of exposed line tripping event due to the hidden failure. The results of the sensitive transmission lines for the IEEE-RTS79 and IEEE-RTS96 are shown in Tables 1 and 2, respectively.

Table 1 shows the results of the sensitive transmission lines obtained based on the critical system cascading collapse for the IEEE-RTS79. For the three case studies of $p_{\mathrm{HF}}$, the $\mu\left(\widehat{P}_{C_{i}}\right)$ was ranked in descending order to identify the sensitive transmission lines that will lead to a critical system cascading collapse. From the results obtained, it was found that the sensitive transmission line 12-13, line 14-16, and line 12-23 provide significantly large value of $\mu\left(\widehat{P}_{C_{i}}\right)$ compared to other lines in the system and this can also be observed in Figure 3.

In Figure 3, the initial tripping of sensitive transmission line 12-13 and line 14-16 leading to a sudden increase of $\mu\left(\widehat{P}_{C_{i}}\right)$ which implies that the system is experiencing a critical cascading collapse. Therefore, major precaution should be given to circumvent from the disconnection of the three sensitive transmission lines which will lead to a critical system cascading collapse. Besides that, the $p_{\mathrm{HF}}=8 \times 10^{-7}$ yields the highest value of $\mu\left(\widehat{P}_{C_{i}}\right)$ for all initial lines tripping compared to the other two probabilities which are $p_{\mathrm{HF}}=1 \times 10^{-12}$ and $p_{\mathrm{HF}}=1 \times 10^{-2}$. This implies that the actual information of cascading collapse events that caused by the protection system hidden failure is important to be taken into account in the $p_{\mathrm{HF}}$ calculation because it will give a significant difference and an accurate result of $\mu\left(\widehat{P}_{C_{i}}\right)$.

Table 2 represents the results of sensitive transmission lines obtained from the analysis of cascading collapse for the
TABLE 1: Estimated average probability of cascading collapse $\mu\left(\widehat{P}_{C_{i}}\right)$ of each initial line tripping due to the effect of hidden failure for IEEE-RTS79.

\begin{tabular}{lccccc}
\hline \multicolumn{2}{c}{$p_{\mathrm{HF}}=1 \times 10^{-12}$} & \multicolumn{2}{c}{$p_{\mathrm{HF}}=8 \times 10^{-7}$} & \multicolumn{2}{c}{$p_{\mathrm{HF}}=1 \times 10^{-2}$} \\
$\begin{array}{l}\text { Initial line } \\
\text { tripping }\end{array}$ & $\begin{array}{c}\mu\left(\widehat{P}_{\mathrm{C}_{i}}\right) \\
\text { (per unit) }\end{array}$ & $\begin{array}{c}\text { Initial line } \\
\text { tripping }\end{array}$ & $\begin{array}{c}\mu\left(\widehat{P}_{\mathrm{C}_{i}}\right) \\
\text { (per unit) }\end{array}$ & $\begin{array}{c}\text { Initial line } \\
\text { tripping }\end{array}$ & $\begin{array}{c}\mu\left(\widehat{P}_{\mathrm{C}_{i}}\right) \\
\text { (per unit) }\end{array}$ \\
\hline $12-13$ & 0.35740 & $12-13$ & 0.35579 & $12-13$ & 0.24756 \\
$14-16$ & 0.22384 & $14-16$ & 0.23058 & $14-16$ & 0.16518 \\
$12-23$ & 0.19249 & $12-23$ & 0.19084 & $12-23$ & 0.14123 \\
$1-5$ & 0.13625 & $3-9$ & 0.18691 & $1-5$ & 0.13625 \\
$3-9$ & 0.13611 & $2-4$ & 0.18120 & $3-9$ & 0.13611 \\
$2-4$ & 0.13417 & $1-5$ & 0.17969 & $2-4$ & 0.13417 \\
$18-21$ & 0.13296 & $17-22$ & 0.17913 & $18-21$ & 0.13296 \\
$17-22$ & 0.13277 & $18-21$ & 0.17856 & $17-22$ & 0.13277 \\
\hline
\end{tabular}

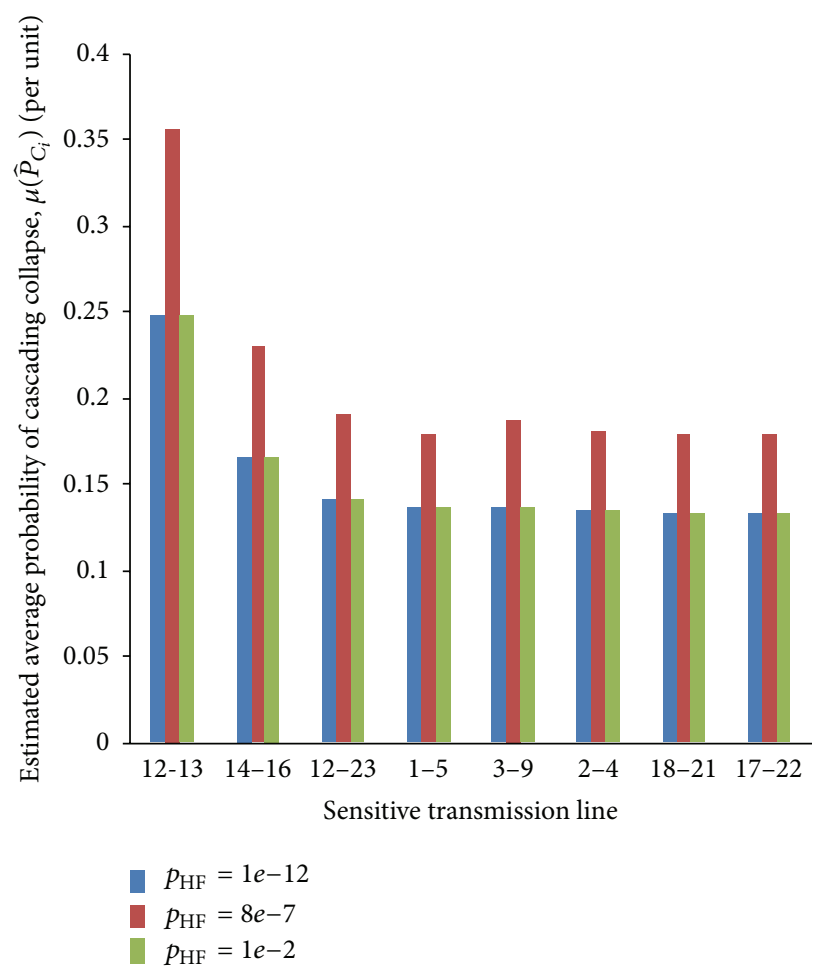

FIGURE 3: Estimated average probability of cascading collapse $\mu\left(\widehat{P}_{C_{i}}\right)$ of each initial line tripping for IEEE RTS-79.

IEEE-RTS96. For the case studies of $p_{\mathrm{HF}}=1 \times 10^{-12}$ and $p_{\mathrm{HF}}=8 \times 10^{-7}$, the initial tripping of sensitive transmission line $318-223$, line $112-113$, and line $113-123$ yields a significantly high value of $\mu\left(\widehat{P}_{C_{i}}\right)$, compared to the initial tripping of other transmission lines. The effect of $p_{\mathrm{HF}}=1 \times 10^{-12}, p_{\mathrm{HF}}=8 \times$ $10^{-7}$, and $p_{\mathrm{HF}}=1 \times 10^{-2}$ can also be observed in Figure 4 whereby critical cascading collapse may occur due to a rapid increase of $\mu\left(\widehat{P}_{C_{i}}\right)$ caused by the initial tripping of the three sensitive transmission lines.

In Figure 4, the initial tripping of sensitive transmission line 318-223, line 112-113, and line 113-123 leads to a sudden increase of $\mu\left(\widehat{P}_{C_{i}}\right)$ and these are referring to the three case 
TABLE 2: Estimated average probability of cascading collapse $\mu\left(\widehat{P}_{C_{i}}\right)$ of each initial line tripping due to the effect of hidden failure for IEEE-RTS96.

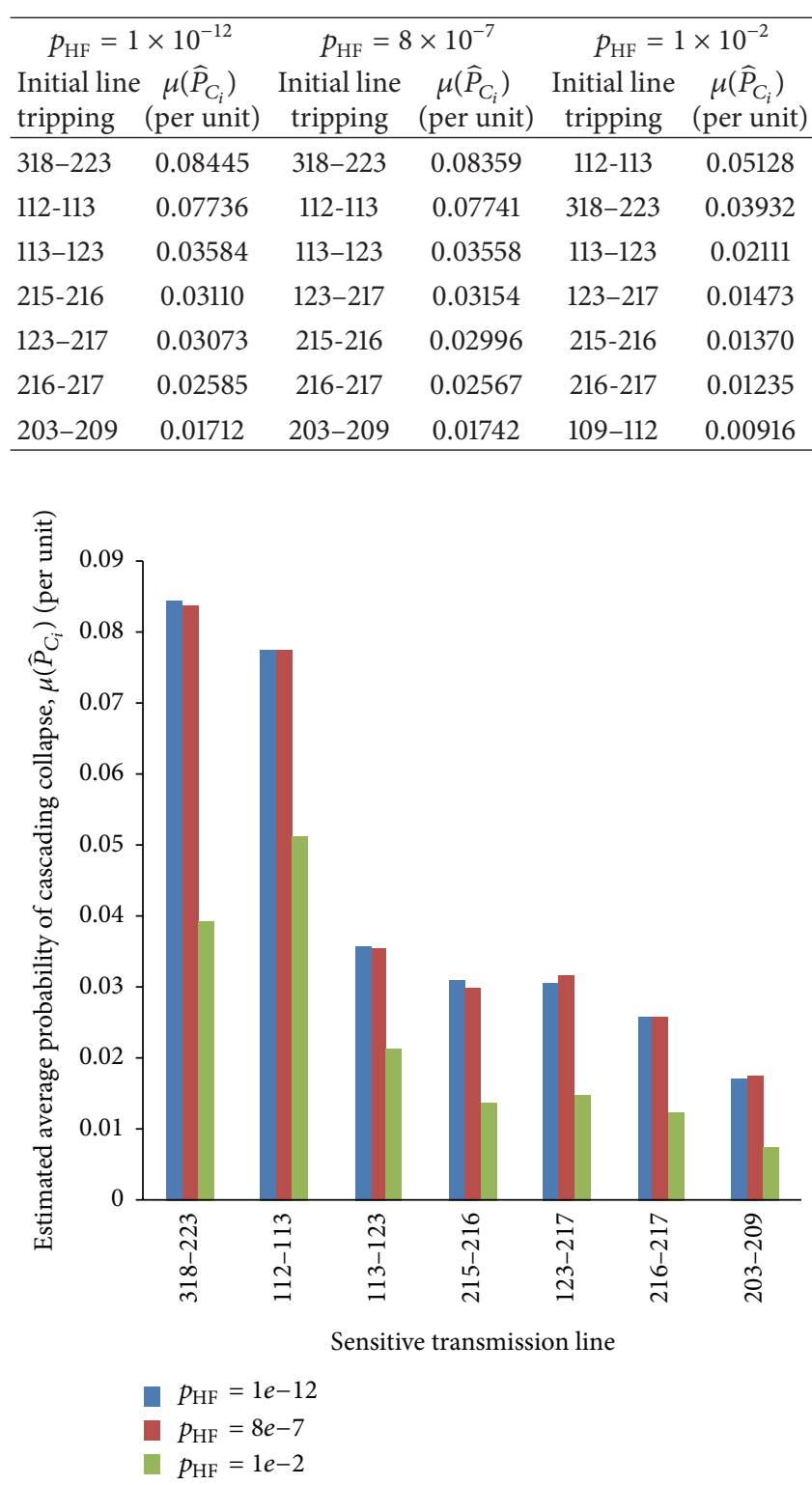

FIGURE 4: Estimated average probability of cascading collapse $\mu\left(\widehat{P}_{C_{i}}\right)$ of each initial line tripping for IEEE RTS-96.

studies of $p_{\mathrm{HF}}$. For the case study of $p_{\mathrm{HF}}=1 \times 10^{-2}$, even though the three sensitive lines are not in a congenial sequence compared to the case studies of $p_{\mathrm{HF}}=1 \times 10^{-12}$ and $p_{\mathrm{HF}}=8 \times 10^{-7}$, it still needs to be given more attention because disconnection on any of the sensitive transmission lines may cause critical cascading collapse of the system.

Based on the results of the sensitive transmission lines obtained in this paper, the proposed approach of cascading collapse is an important and useful method that can be used by the utility which will facilitate them in providing the best planning decision to prevent the critical cascading collapse from happening.
TABLE 3: Estimated average probability of system cascading collapse, $\mu\left(\widehat{P}_{C_{l}}\right)$ for IEEE-RTS79.

\begin{tabular}{lccc}
\hline $\begin{array}{l}\text { Total loading } \\
\text { condition } \\
(\%)\end{array}$ & \multicolumn{3}{c}{$\begin{array}{c}\text { Estimated average probability of system } \\
\text { cascading collapse, } \mu\left(\widehat{P}_{C_{l}}\right) \text { (per unit) }\end{array}$} \\
\hline 150 & $p_{\mathrm{HF}}=1 \times 10^{-12}$ & $p_{\mathrm{HF}}=8 \times 10^{-7}$ & $p_{\mathrm{HF}}=1 \times 10^{-2}$ \\
160 & 0.00723221 & 0.00713164 & 0.00460562 \\
170 & 0.00534533 & 0.00537957 & 0.00332890 \\
180 & 0.01705183 & 0.01715082 & 0.01068545 \\
190 & 0.12649106 & 0.12658082 & 0.07700219 \\
200 & 0.15640375 & 0.15650766 & 0.10102151 \\
210 & 0.17128861 & 0.17108597 & 0.11174890 \\
220 & 0.21116515 & 0.21003825 & 0.14113007 \\
230 & 0.19935382 & 0.19852302 & 0.13679530 \\
240 & 0.23980806 & 0.24100823 & 0.17056760 \\
\hline & 0.50190445 & 0.50233278 & 0.42771526 \\
\hline
\end{tabular}

4.2. Criticality of System Cascading Collapse due to the Effect of Hidden Failure and Severe Total Loading Condition. In this section, the estimated average probability of cascading collapse $\mu\left(\widehat{P}_{C_{l}}\right)$ is used in obtaining the severity of total loading condition which may lead to a critical system cascading collapse. From the three different case studies of hidden failure which are $p_{\mathrm{HF}}=8 \times 10^{-7}, p_{\mathrm{HF}}=1 \times 10^{-12}$, and $p_{\mathrm{HF}}=$ $1 \times 10^{-2}$, the results for the $\mu\left(\widehat{P}_{C_{l}}\right)$ are calculated and it is tabulated in Tables 3 and 4 corresponding to the IEEE-RTS79 and IEEE-RTS96, respectively. By referring to Table 3, the $\mu\left(\widehat{P}_{C_{l}}\right)$ is also obtained in accordance with the total loading condition increased from $150 \%$ to $240 \%$ for the IEEE-RTS79. The results shown in Table 3 are also depicted in Figure 5.

In conjunction to the three case studies of hidden failure, the $\mu\left(\widehat{P}_{C_{l}}\right)$ varies as the total loading condition increased by $10 \%$. In addition, an upward trend of $\mu\left(\widehat{P}_{C_{l}}\right)$ is quite significant for all the three case studies when the total loading condition increased above $170 \%$. Moreover, the highest value of $\mu\left(\widehat{P}_{C_{l}}\right)$ is obtained when the total loading condition increased above $230 \%$. This information can be useful to the utility for estimating the maximum allowable level of total loading condition before the system is afflicted with the highest risk of cascading collapse. There are no significant difference between the $\mu\left(\widehat{P}_{C_{l}}\right)$ obtained on the $p_{\mathrm{HF}}=8 \times 10^{-7}$ and $p_{\mathrm{HF}}=1 \times 10^{-12}$. For an example, the results of $\mu\left(\widehat{P}_{\mathrm{C}_{l}}\right)=$ 0.50190445 and $\mu\left(\widehat{P}_{C_{l}}\right)=0.50233278$ are relatively similar and these are referring to the $p_{\mathrm{HF}}=1 \times 10^{-12}$ and $p_{\mathrm{HF}}=8 \times$ $10^{-7}$, respectively, obtained at $240 \%$ increase of total loading condition.

Therefore, any $p_{\mathrm{HF}}$ value that is lower than $p_{\mathrm{HF}}=1 \times 10^{-12}$ will produce similar result of $\mu\left(\widehat{P}_{C_{1}}\right)$ as in the case study of $p_{\mathrm{HF}}=8 \times 10^{-7}$. However, the two $p_{\mathrm{HF}}$ values are providing the results of $\mu\left(\widehat{P}_{C_{l}}\right)$ with higher risk compared to the $\mu\left(\widehat{P}_{C_{l}}\right)$ obtained based on $p_{\mathrm{HF}}=1 \times 10^{-2}$. This indicates that it important to choose the correct value of $p_{\mathrm{HF}}$ in order to 


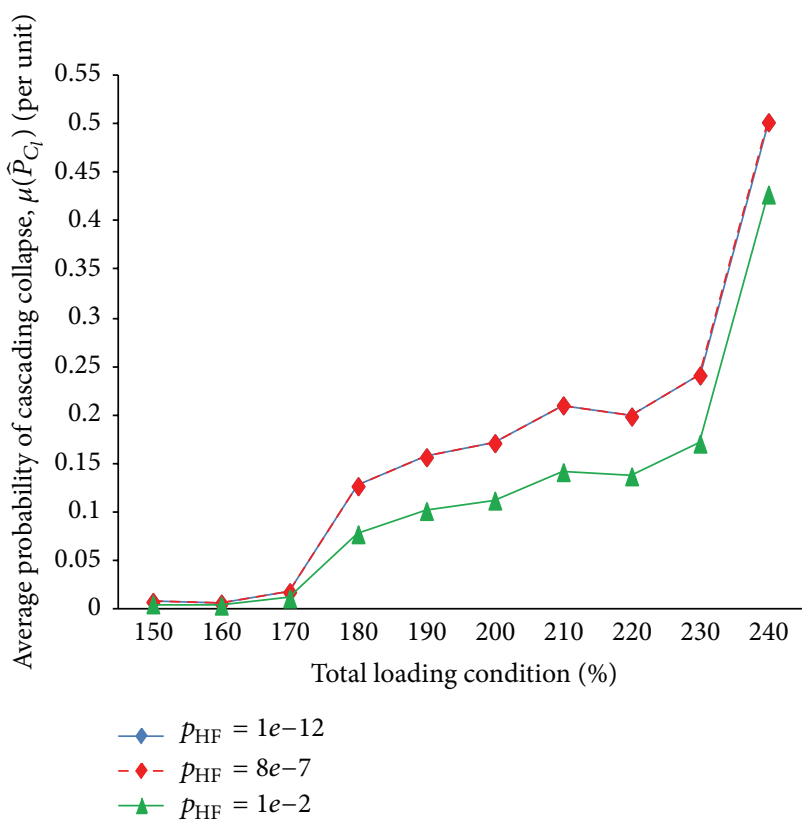

FIGURE 5: Estimated average probability of cascading collapse $\mu\left(\widehat{P}_{C_{l}}\right)$ at every increased of total loading condition for IEEE-RTS79.

TABLE 4: Estimated average probability of system cascading collapse, $\mu\left(\widehat{P}_{C_{l}}\right)$ for IEEE-RTS96.

\begin{tabular}{lccc}
\hline \multirow{2}{*}{$\begin{array}{l}\text { Total loading } \\
\text { Condition }(\%)\end{array}$} & \multicolumn{3}{c}{ Estimated average probability of system } \\
& cascading collapse, $\mu\left(\widehat{P}_{\mathrm{C}_{l}}\right)$ (per unit) \\
& $p_{\mathrm{HF}}=1 \times 10^{-12}$ & $p_{\mathrm{HF}}=8 \times 10^{-7}$ & $p_{\mathrm{HF}}=1 \times 10^{-2}$ \\
\hline 130 & 0.00019344 & 0.00019056 & 0.00000084 \\
140 & 0.00025224 & 0.00025606 & 0.00000217 \\
150 & 0.00008205 & 0.00008217 & 0.00000217 \\
160 & 0.00010118 & 0.00010436 & 0.00002218 \\
170 & 0.00003173 & 0.00003118 & 0.00000583 \\
180 & 0.00001718 & 0.00001708 & 0.00000285 \\
190 & 0.00005894 & 0.00005892 & 0.00000930 \\
200 & 0.00271678 & 0.00271430 & 0.00069031 \\
210 & 0.02335387 & 0.02333729 & 0.00923196 \\
220 & 0.08822664 & 0.08820120 & 0.05050363 \\
\hline
\end{tabular}

obtain an accurate estimated average probability of system cascading collapse, $\mu\left(\widehat{P}_{C_{l}}\right)$.

Table 4 illustrates the result of $\mu\left(\widehat{P}_{C_{1}}\right)$ that is obtained by increasing the total loading condition from $130 \%$ to $220 \%$ for IEEE-RTS96. For this case study, the analysis of cascading collapse was performed based on the probability of hidden failure that is $p_{\mathrm{HF}}=8 \times 10^{-7}, p_{\mathrm{HF}}=1 \times 10^{-12}$, and $p_{\mathrm{HF}}=$ $1 \times 10^{-2}$. The results tabulated in Table 4 are also illustrated in Figure 6. It is obvious that the results of $\mu\left(\widehat{P}_{C_{1}}\right)$ are rather small possibly due to a stable system condition assisted by a large number of generating units and transmission lines. Even though the $\mu\left(\widehat{P}_{C_{1}}\right)$ is relatively small, it cannot not be ignored because its impact to the power system could be disastrous.

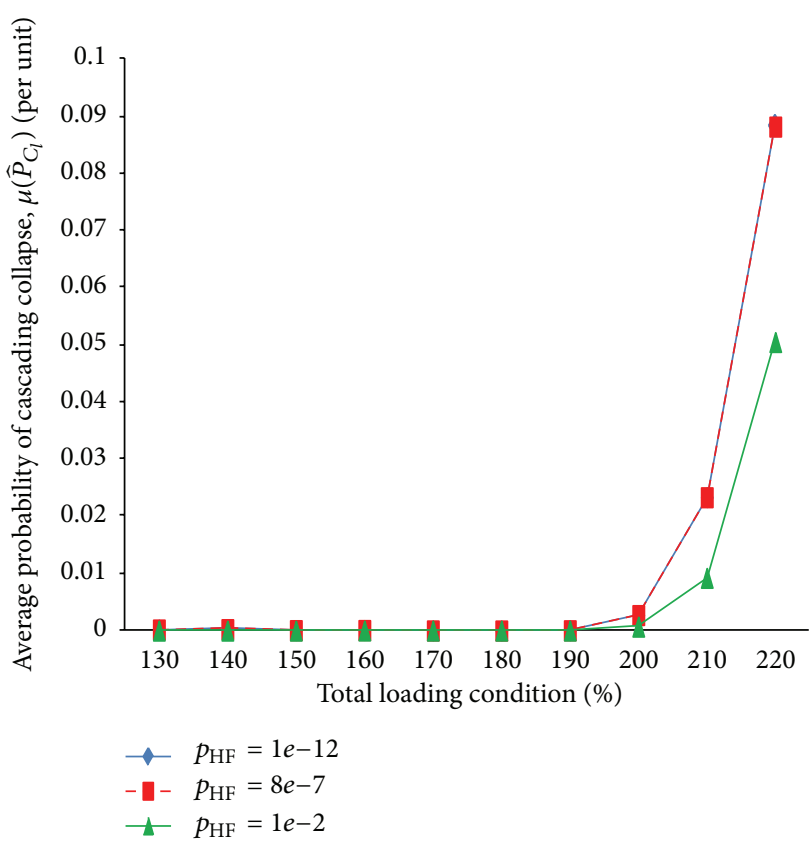

FIGURE 6: Estimated average probability of cascading collapse $\mu\left(\widehat{P}_{C_{l}}\right)$ at every increased of total loading condition for IEEE-RTS96.

A rapid increase of $\mu\left(\widehat{P}_{C_{1}}\right)$ can be seen for all the three case studies when the total loading condition is increased beyond $200 \%$. It will continue to increase significantly until it reaches to the highest $\mu\left(\widehat{P}_{C_{1}}\right)$ due to the $220 \%$ of total loading condition. This information is useful to identify which total loading condition agitates to the criticality of a system cascading collapse. From the three different case studies of $p_{\mathrm{HF}}, p_{\mathrm{HF}}=8 \times 10^{-7}$ and $p_{\mathrm{HF}}=1 \times 10^{-12}$ provide higher risk compared to the $\mu\left(\widehat{P}_{\mathrm{C}_{l}}\right)$ obtained based on $p_{\mathrm{HF}}=$ $1 \times 10^{-2}$. This points out that it is significant to select the accurate value of $p_{\mathrm{HF}}$ in order to obtain an accurate estimated average probability of system cascading collapse, $\mu\left(\widehat{P}_{C_{l}}\right)$.

A detailed analysis of cascading collapse has been carried out on the IEEE-RTS79 and IEEE-RTS9 6 by taking into consideration the three different values of probability of hidden failure which are $p_{\mathrm{HF}}=8 \times 10^{-7}, p_{\mathrm{HF}}=1 \times 10^{-12}$, and $p_{\mathrm{HF}}=1 \times 10^{-2}$. From the results obtained, it is important to determine the accurate value of probability of hidden failure, $p_{\mathrm{HF}}$, since this will significantly affect the results of estimated average probability of cascading collapse, $\mu\left(\widehat{P}_{C_{1}}\right)$, and the criticality of system cascading collapse prior to a severe total loading condition.

4.3. Performance Comparison between the Cascading Collapse Methods. Comparative study was performed on the results of sensitive transmission lines determined by using the proposed method and fault chain theory discussed in [11]. It is worthwhile to mention that an initial tripping of sensitive transmission line will instigate to a critical system cascading collapse. The robustness of both methods in the sensitive transmission line determination is tested on the IEEE 14-bus system which comprised of 20 transmission 
TABLE 5: Results of each initial line tripping for IEEE 14-bus system using the proposed method and fault chain theory.

\begin{tabular}{|c|c|c|c|}
\hline \multicolumn{2}{|c|}{ Proposed method } & \multicolumn{2}{|c|}{ Fault chain theory [11] } \\
\hline $\begin{array}{l}\text { Initial line } \\
\text { tripping }\end{array}$ & $\mu\left(\widehat{P}_{C_{i}}\right)$ & $\begin{array}{l}\text { Initial line } \\
\text { tripping }\end{array}$ & $\begin{array}{c}\text { Vulnerability } \\
\text { index }\end{array}$ \\
\hline $4-5$ & 0.9587 & $1-2$ & 0.1838 \\
\hline $2-3$ & 0.6274 & $2-4$ & 0.1838 \\
\hline $2-4$ & 0.5546 & $4-7$ & 0.1134 \\
\hline $1-2$ & 0.5322 & $6-13$ & 0.0644 \\
\hline $9-14$ & 0.5068 & $1-5$ & 0.0570 \\
\hline $9-10$ & 0.5021 & $9-14$ & 0.0550 \\
\hline $4-9$ & 0.4867 & $6-11$ & 0.0515 \\
\hline $2-5$ & 0.4577 & $3-4$ & 0.0478 \\
\hline 7-9 & 0.4266 & $2-3$ & 0.0447 \\
\hline $7-8$ & 0.3905 & $6-12$ & 0.0344 \\
\hline $1-5$ & 0.3536 & $4-5$ & 0.0275 \\
\hline $10-11$ & 0.3371 & $12-13$ & 0.0275 \\
\hline $4-7$ & 0.3295 & $9-10$ & 0.0206 \\
\hline $3-4$ & 0.3287 & $2-5$ & 0.0172 \\
\hline $12-13$ & 0.3193 & $4-9$ & 0.0172 \\
\hline $6-11$ & 0.3101 & $5-6$ & 0.0172 \\
\hline $6-12$ & 0.2873 & $10-11$ & 0.0172 \\
\hline $6-13$ & 0.2598 & $13-14$ & 0.0172 \\
\hline $5-6$ & 0.2080 & $7-8$ & 0.0129 \\
\hline $13-14$ & 0.1590 & $7-9$ & 0.0103 \\
\hline
\end{tabular}

lines, 5 generating units, and 11 load buses. The total load for the system is $259 \mathrm{MW}$ and the total generation capacity is $272 \mathrm{MW}$. The process involved in the proposed method is comparatively similar with the fault chain theory wherein the subsequent tripping of transmission line is executed until the system instability occurs. Eventually, major tripping of transmission line may lead to an inadequate amount of total generation capacity required by the total load demand and this will agitate to system instability. The disadvantage of fault chain theory is that it does not consider the incorrect line tripping caused by protection relay hidden failure. This may yield to an inaccurate result of sensitive transmission lines.

Table 5 and Figure 7 present the results of initial line tripping ranked in descending order according to $\mu\left(\widehat{P}_{C_{l}}\right)$ and vulnerability index determined by using the proposed method and fault chain theory [11], respectively. It is obvious that the initial transmission line tripping was ranked differently by both methods. Hence, the obtained sensitive transmission lines will be different for both methods. The proposed method with hidden failure provides the most sensitive transmission line 4-5 which is not similar to the most sensitive transmission line 1-2 and line 2-4 determined by the fault chain theory. From the results, it is obvious that the proposed method provides the most sensitive transmission line 4-5 based on the largest value of $\mu\left(\widehat{P}_{C_{i}}\right)$ compared to other lines in the system. On the other hand, the fault chain theory provides the most sensitive transmission line 1-2 and line 2-4

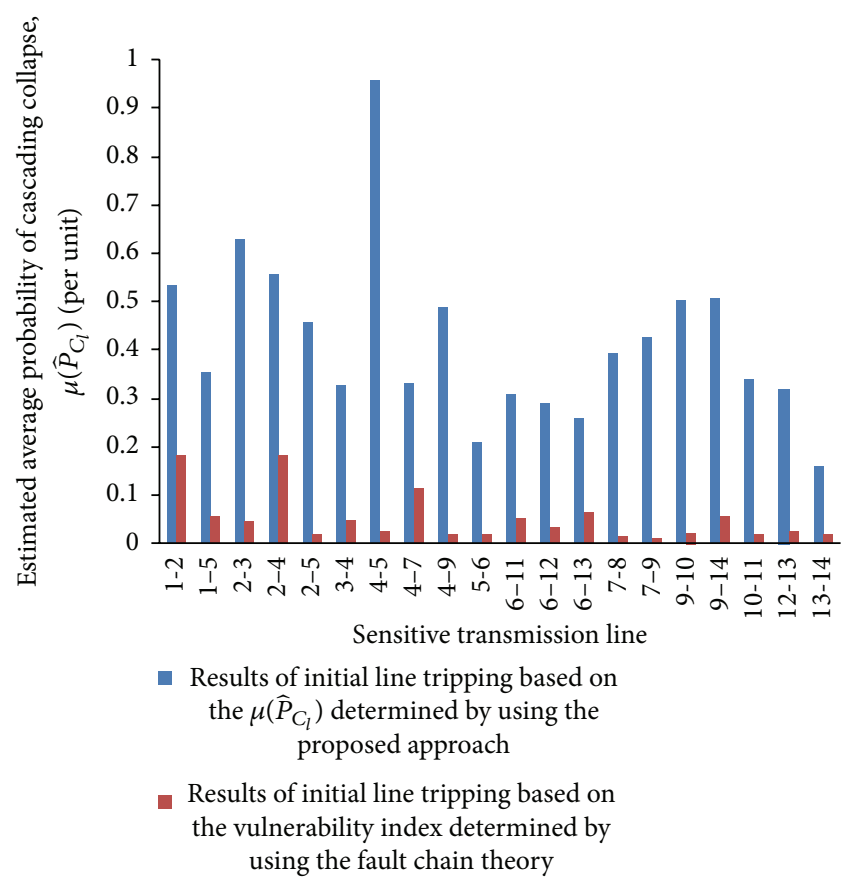

FIGURE 7: Comparative results of the sensitive transmission lines for IEEE 14-bus system.

based on the largest value of vulnerability index compared to the rest of the transmission lines.

However, the proposed method with hidden failure accentuates to a clearer picture on the results of $\mu\left(\widehat{P}_{C_{i}}\right)$ which is larger and explicit compared to the results of vulnerability index determined by the fault chain theory. The proposed method with hidden failure will assist the researcher to a much easier way in determining the sensitive transmission lines which is based on the $\mu\left(\widehat{P}_{C_{i}}\right) \geq 50 \%$ and vice versa. Therefore, the transmission line $4-5$, line $2-3$, line $2-4$, line $1-2$, and line $9-14$ are considered sensitive determined by the proposed method with hidden failure.

This implies that the inclusion of hidden failure in the proposed method provides more accurate results of sensitive transmission lines compared to the fault chain theory which does not consider the hidden failure. In particular, the historical information of incorrect line tripping caused by hidden failure is represented by $p_{\mathrm{HF}}$ wherein it is the main contribution which improves the performance of the proposed method in providing more accurate result of sensitive transmission lines. According to [16], cascaded tripping of transmission lines is the main cause to a system blackout which may disrupt the economic and social life of nation in a country. As a result, it is crucially important for the utility and power system planner to identify the accurate sensitive transmission lines in order to circumvent from disastrous impact of system cascading collapse.

\section{Conclusions}

The escalating number of critical cascading collapse happened recently has revealed that there is an urgent need 
for new techniques required by the system planning and operation. The critical system cascading collapse usually can happen just by an initial tripping event of a transmission line. This paper has discussed the estimated average probability of cascading collapse, evaluated by assuming that each transmission line has a different load-dependent probability of incorrect tripping due to hidden failure. In this paper, the estimated average probability of cascading collapse was determined based on three different case studies of hidden failure probability. The results have shown that it is imperative to select an accurate probability of hidden failure as it has a significant effect on the result of estimated average probability of cascading collapse. The evaluation of critical cascading events due to hidden failure should be carried out periodically in the power system operation and planning in order to avert the power system from any kind of catastrophic events. The estimated average probability of cascading collapse was also analyzed to determine the sensitive initial transmission lines tripping and also the severity of total loading condition. For that reason, precaution and necessary actions should be made by the utility and power system planner to ensure that severe total loading condition does not occur and that sensitive transmission lines are well preserved or maintained in order to avoid catastrophic impact on system cascading collapse. Comparison with fault chain theory has proven that the proposed method with hidden failure provides more accurate result of sensitive transmission lines.

\section{Acronym}

OPF: Optimal power flow.

\section{Notation}

$p_{\mathrm{HF}}$ : Probability of exposed line incorrect tripping caused by hidden failure

E: Total number of cascading collapse events due to hidden failure

$y$ : Total number of years when the events of cascading collapse occur

$d$ : Total number of days in a year, that is, 365 days

$h$ : Total number of hours in a day, that is, 24 hours

$m$ : Total number of minutes in an hour, that is, 60 minutes

$s:$ Total number of seconds in a minute, that is, 60 seconds

$P_{c j}$ : Conditional probability of tripping in state $j$

$P_{c i}$ : Product of tripping events probability

$p_{H_{j}}$ : Probability of exposed transmission line encountering the random tripping event in state $j$

$q_{H_{j}}$ : Probability of the exposed transmission line not encountering the random tripping event in state $j$

NJ: Total number of system state at initial tripping, $i$
K: $\quad$ Total number of iterations to perform the random tripping

$L: \quad$ Total number of steps for the increase of total loading condition

I: $\quad$ Total number of transmission line in the system

$\mu\left(\widehat{P}_{C_{i}}\right)$ : Estimated average probability of cascading collapse used to identify the sensitive transmission line contributing to a critical system cascading collapse

$\mu\left(\widehat{P}_{C_{1}}\right)$ : Estimated average probability of system cascading collapse used to identify the criticality of system cascading collapse prior to a severe total loading condition.

\section{Acknowledgments}

The authors would like to thank the Research Management Institute (RMI), Universiti Teknologi MARA, Malaysia, and the Ministry of Higher Education (MOHE), Malaysia, through research grant 600-RMI/ERGS5/3(18/2012) for the financial support of this research. The authors also would like to express their sincere gratitude to Professor Mahmud Fotuhi Firuzabad from Sharif University of Technology, Iran, for his continuous support in completing this research.

\section{References}

[1] H. Pidd, "India blackouts leave 700 million without power," http://www.guardian.co.uk/world/2012/jul/31/india-blackoutelectricity-power-cuts.

[2] A. S. Bakshi, A. Velayutham, S. C. Srivastava et al., "Report of the Enquiry Committee on Grid Disturbance in Northern Region on 30th July 2012 and in Northern, Eastern \& North-Eastern Region on 31st July 2012," New Delhi, India, 2012.

[3] FERC and NERC, "Arizona-Southern California Outages on September 8, 2011. Causes and Recommendations," 2012.

[4] M. Vaiman, K. Bell, Y. Chen et al., "Risk assessment of cascading outages: methodologies and challenges," IEEE Transactions on Power Systems, vol. 27, no. 2, pp. 631-641, 2012.

[5] I. Dobson, J. McCalley, and C. C. Liu, Fast Simulation, Monitoring and Mitigation of Cascading Failure, Power Systems Engineering Research Center, 2010.

[6] I. Dobson, "Estimating the propagation and extent of cascading line outages from utility data with a branching process," IEEE Transactions on Power Systems, vol. 27, no. 4, pp. 2146-2155, 2012.

[7] B. A. Carreras, D. E. Newman, and I. Dobson, "Determining the vulnerabilities of the power transmission system," in Proceedings of the 45th Hawaii International Conference on System Sciences (HICSS '12), pp. 2044-2053, January 2012.

[8] G. Chen, Z. Y. Dong, D. J. Hill, G. H. Zhang, and K. Q. Hua, "Attack structural vulnerability of power grids: a hybrid approach based on complex networks," Physica A, vol. 389, no. 3, pp. 595-603, 2010.

[9] Z. Shi, L. Shi, Y. Ni, L. Yao, and M. Bazargan, "Identifying chains of events during power system cascading failure," in Proceedings of the Asia-Pacific Power and Energy Engineering Conference (APPEEC '11), March 2011. 
[10] S.-P. Wang, A. Chen, C.-W. Liu, C.-H. Chen, and J. Shortle, "Rare-event splitting simulation for analysis of power system blackouts," in Proceedings of the IEEE Power and Energy Society General Meeting, July 2011.

[11] A. Wang, Y. Luo, G. Tu, and P. Liu, "Vulnerability assessment scheme for power system transmission networks based on the fault chain theory," IEEE Transactions on Power Systems, vol. 26, no. 1, pp. 442-450, 2011.

[12] J. Chen, J. S. Thorp, and I. Dobson, "Cascading dynamics and mitigation assessment in power system disturbances via a hidden failure model," International Journal of Electrical Power and Energy Systems, vol. 27, no. 4, pp. 318-326, 2005.

[13] N. A. Salim, M. M. Othman, I. Musirin, and M. S. Serwan, "Cascading collapse assessment considering hidden failure," in Proceedings of the 1st International Conference on Informatics and Computational Intelligence (ICI '11), pp. 318-323, December 2011.

[14] C. Grigg and P. Wong, "The IEEE reliability test system-1996 a report prepared by the reliability test system task force of the application of probability methods subcommittee," IEEE Transactions on Power Systems, vol. 14, no. 3, pp. 1010-1020, 1999.

[15] P. M. Subcommittee, "IEEE reliability test system," IEEE Transactions on Power Apparatus and Systems, vol. 98, no. 6, pp. 20472054, 1979.

[16] M. Bruch, V. Munch, M. Aichinger, M. Kuhn, M. Weymann, and G. Schmid, "Power blackout risks. Risk management options. Emerging risk initiative-position paper," November 2011. 


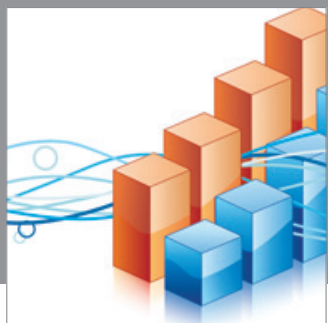

Advances in

Operations Research

mansans

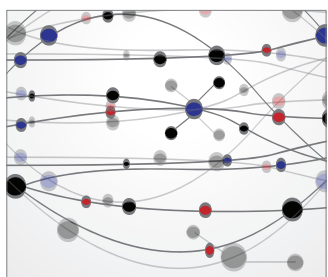

The Scientific World Journal
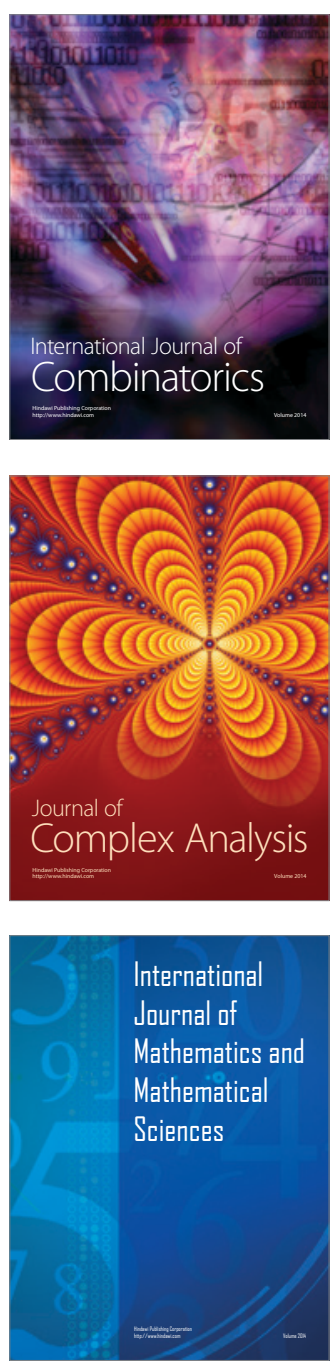
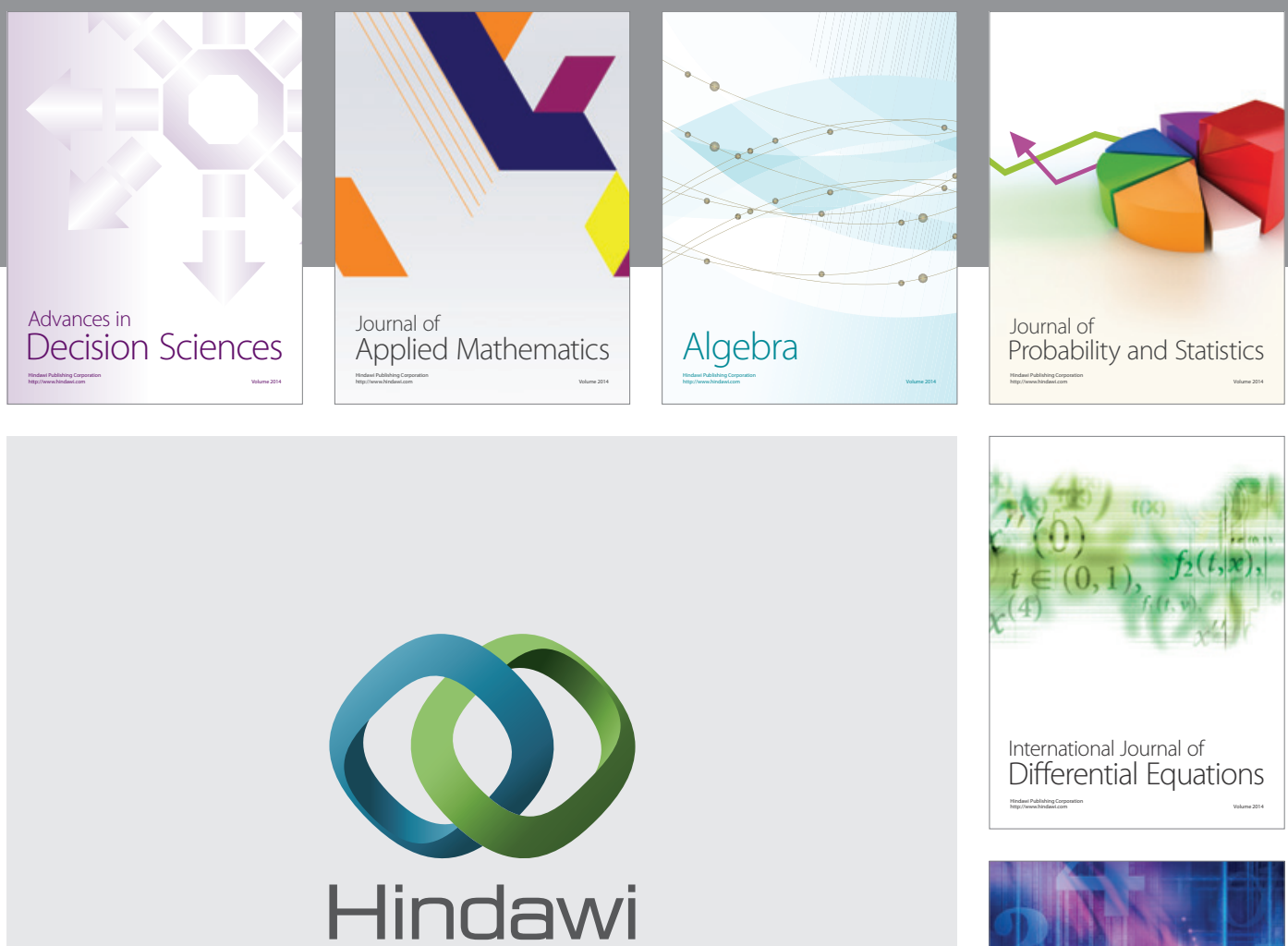

Submit your manuscripts at http://www.hindawi.com
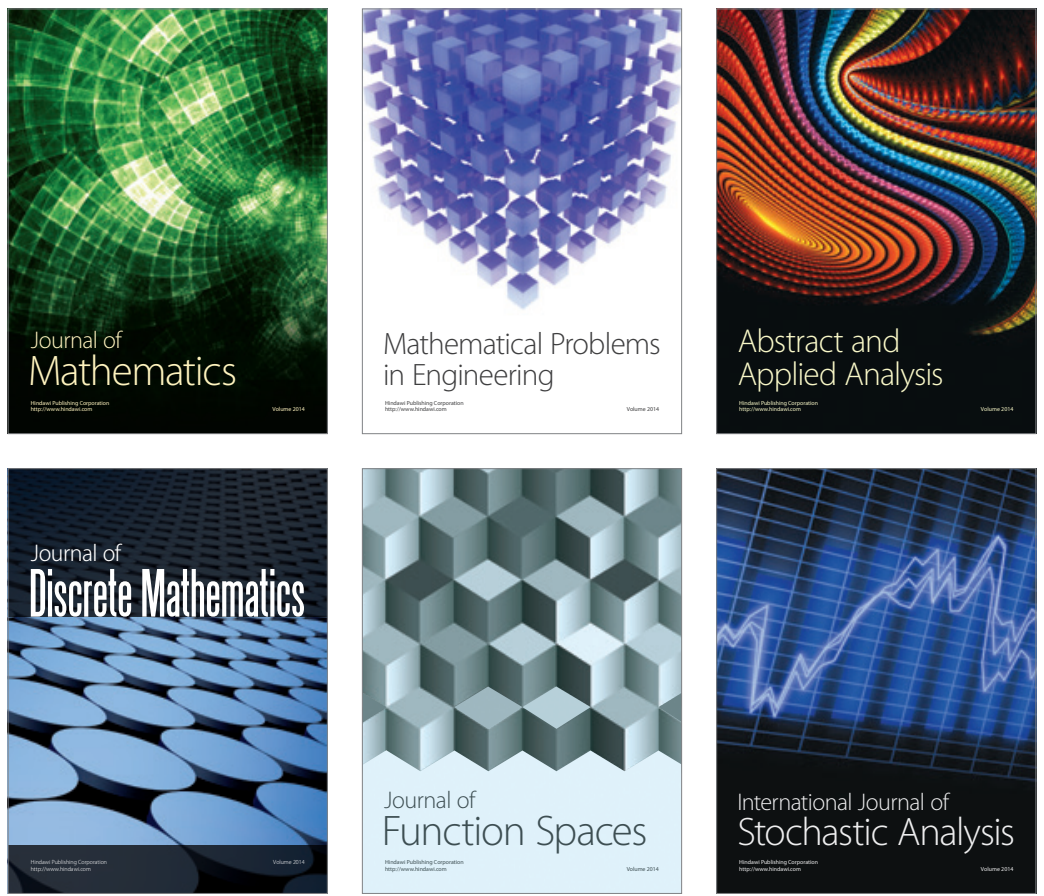

Journal of

Function Spaces

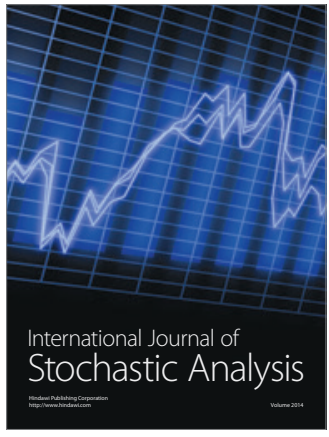

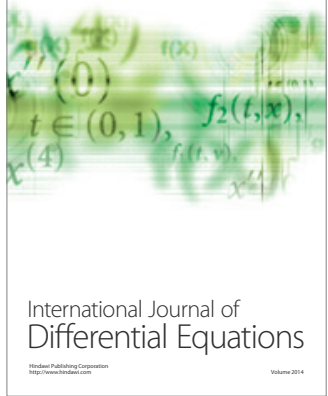
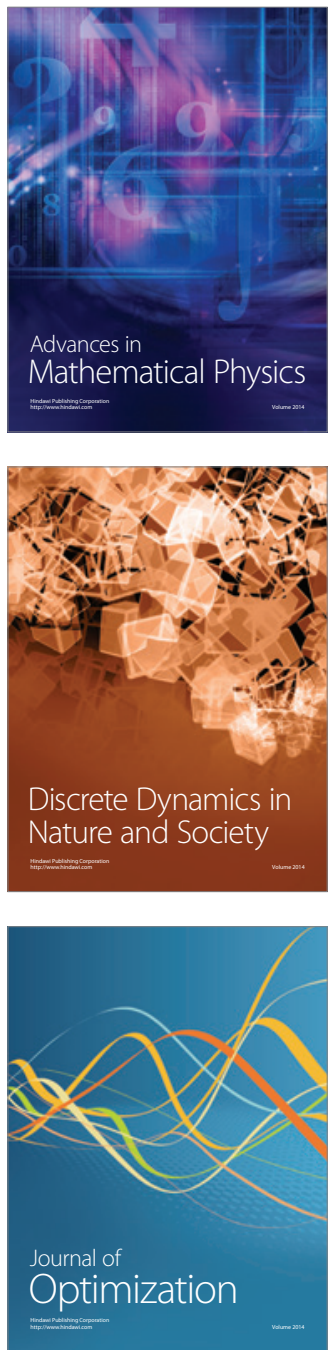